\title{
In vitro validation of right ventricular volume measurement by three dimensional echocardiography
}

\author{
Michael Vogel, Paul A White, Andrew N Redington
}

\begin{abstract}
Objective-Evaluation of ability of three dimensional echocardiography to accurately assess right ventricular volumes in vitro.
\end{abstract}

Methods-Silicone casts of normal human right ventricles were examined. Each was filled with three different volumes of water to yield 15 different measurements. The casts were examined in a waterbath with three dimensional echocardiography using a $7.5 \mathrm{MHz}$ ultrasound probe mounted in a scan frame. It was steered by a stepper motor, which moved the probe in steps of $0.25 \mathrm{~mm}$ over a distance of $5.9 \mathrm{~cm}$ inside the frame, acquiring an image at each step. 236 parallel slices of the cast were thus obtained, forming the three dimensional dataset. The longest axis of the right ventricular volume was defined and the area of perpendicular $1 \mathrm{~mm}$ thick slices was outlined manually to calculate the area of each slice. This was multiplied by the slice thickness to obtain the volume of each slice; the respective volumes were added to obtain the volume of the whole cast. Results-The casts had a median volume of 31.1 (23) $\mathrm{ml}$ (range 15-100); three dimensional echocardiography gave a median volume of $29.0(21 \cdot 7) \mathrm{ml}(15 \cdot 7$ $91 \cdot 7)$. Interobserver variability was $4 \cdot 5 \%$ $(0.4 \%-13 \cdot 6 \%)$ and intraobserver variability $4 \cdot 3 \% \quad(0 \cdot 2 \%-9 \cdot 3 \%)$. Correlation between real cast volumes and volumes measured by three dimensional echocardiography was $0.99(y=1.08 x-0.16)$ with an SEE of $2.7 \mathrm{ml}$. Limits for agreement between methods ranged from $-3.1 \mathrm{ml}$ to $8.3 \mathrm{ml}$. In 14 of the $15 \mathrm{mea}-$ surements, volume by three dimensional echocardiography was smaller than real volume, with the mean difference being $7 \cdot 4 \%(2 \cdot 8 \%-19 \cdot 5 \%)$. This may be due to the thickening of surfaces of structures when imaged by ultrasonography. Conclusion-Right ventricular volumes can accurately be determined by three dimensional echocardiography.

(Br Heart f 1995;74:460-463)

Keywords: echocardiography; volume assessment
Interest in assessing right ventricular dimensions and volumes by various imaging techniques, including echocardiography, is long standing. ${ }^{12}$ Numerous studies have shown that accurate assessment of right ventricular volume is difficult as simple geometric formulas such as Simpson's rule ${ }^{34}$ or an area and length calculation cannot be reliably used. Recently three dimensional echocardiography has become available ${ }^{56}$ and has been successfully applied for the assessment of left ventricular volumes. ${ }^{7}$ Three dimensional echocardiography has also been used to assess right ventricular volumes by determining the volumes of a balloon inserted into right ventricles in an in vivo animal study. ${ }^{8}$ This study, however, did not take into account the rough irregular endocardial surface, as the right ventricular trabeculations were smoothened out by the balloon. We used casts of right ventricles from humans with irregular interior surfaces to assess the accuracy of three dimensional echocardiography in vitro.

Materials and methods ECHOCARDIOGRAPHY EQUIPMENT

A commercially available Vingmed 800 (Vingmed, Horten, Norway) annular array sector scanner was interfaced with a Tomtec (Tomtec, Munich, Germany) computer, which generates three dimensional reconstructions derived from cross sectional images. To acquire multiple sequential parallel cross sections of the heart, the transducer is mounted inside a scan frame. It moves longitudinally over a distance of $5.9 \mathrm{~cm}$ in steps of $0.25 \mathrm{~mm}$. Its movement is controlled by a stepper motor. At each step a tomographic slice of the heart is recorded, and a dataset of 236 parallel slices of the heart is acquired by controlled movement of the transducer from the apex of the heart to the outflow tracts. ${ }^{6}$ In this study we positioned the external scan frame containing the transducer on a tripod placed over a bucket filled with water. To optimise the quality of the image the temperature of the water was kept between $35^{\circ} \mathrm{C}$ and $40^{\circ} \mathrm{C} .{ }^{9} \mathrm{~A}$ nonechogenic fishnet was used to position the right ventricular cast inside the bucket. The distance between the transducer and the casts in the bucket was between $3 \mathrm{~cm}$ and $5 \mathrm{~cm}$, depending on the size of the cast. 
After completion of data acquisition, the spatially aligned multiple sequential cross sectional images were digitally reformatted and subsequently stored as a volume element, or voxel. Data were stored on a $48633 \mathrm{MHz}$ personal computer with a $500 \mathrm{MB}$ hard disk; they were subsequently archived on a removable $600 \mathrm{MB}$ laser disk. ${ }^{10}$ The stored images were reviewed in a cross sectional display like the one used in conventional cross sectional echocardiography. Measurement of volume is based on summing the volumes of multiple slices. Firstly, the longest axis of the right ventricular volume was defined in the appropriate cross section of the dataset. Along the long axis, perpendicular $1 \mathrm{~mm}$ thick slices were displayed as a cross sectional image on the monitor. These were then manually outlined to calculate the area of each slice. This was multiplied with the slice thickness to obtain the volume of each slice; the respective volumes were added to obtain the volume of the whole cast. The traced borders of the respective right ventricular slices were displayed in a wire frame format (fig 1).

\section{CASTS}

We examined five normal acrylic resin casts of the right ventricle obtained at necropsy. After the hearts had been prepared by oversewing both atrioventricular valves, the right and left ventricles were distended to a filling pressure of $10 \mathrm{~cm}$ of resin (equivalent to $8 \mathrm{~mm} \mathrm{Hg}$ ) and $13 \mathrm{~cm}$ of resin (equivalent to $10 \mathrm{~mm} \mathrm{Hg}$ ) respectively to maintain normal orientation of the interventricular septum. Secondly, the hardened resin cast was dissected from the right ventricle. After that a hollow model with a uniform wall thickness of 4-5 mm was constructed out of silicone (Cosmesil), by covering the surface of the solid acrylic model. A valve was inserted into the silicone model on top of the right ventricular outflow tract without distorting the geometry, so that different amounts of fluid could be injected.

Each cast was filled with three arbitrarily chosen different volumes of water injected through this valve. The largest of these volumes distended the cast and a little water leaked out. To be consistent, the accurate

\footnotetext{
Figure 1 Wire frame format of a right ventricular cast constructed out of the manually
outlined multiple slices of the dataset.
}

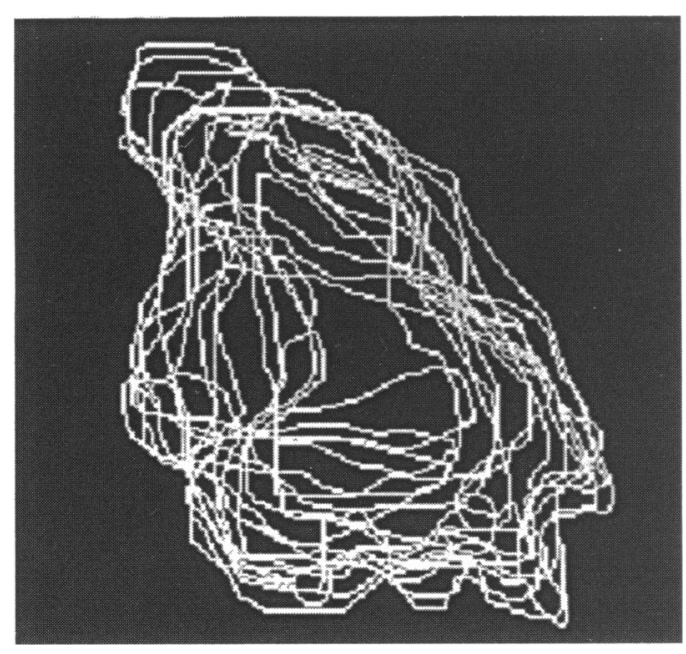

volume of each cast was considered to be the volume of water that was withdrawn from the cast immediately after the cast had been imaged, a procedure that took less than 20 seconds. This withdrawal volume was noted by one observer (PAW) and not told to the other observer (MV) before MV measured the cast volume; MV thus had no knowledge of the true volume of the cast during the measurement.

In addition, we determined right ventricular volume in a heart with normal intracardiac anatomy from the collection of the National Heart and Lung Institute. We closed the tricuspid valve with glue and then injected water through the pulmonary valve until the right ventricle was filled. We then closed the pulmonary valve. We put this heart in the same waterbath and imaged it in the same way as the casts.

\section{STATISTICAL ANALYSIS}

Results of right ventricular volume measurements were compared with the true volumes by simple linear regression analysis. The difference between measured and true volume was expressed as a percentage by dividing the difference by the value of the true volume. Limits for agreement were calculated according to the formulas of Bland and Altman. ${ }^{11}$ Intraobserver variability was assessed by calculating the standard deviation of the differences between repeated measurements of 10 casts; intraobserver variability was similarly determined by calculating the differences between the volume measurements of five casts performed by two independent observers.

\section{Results}

We acquired good quality echocardiographic pictures of all casts. To calculate the volume of a cast took between 12 and 18 minutes, depending on the size of the cast. This time was in addition to the acquisition time of 20 seconds and the three minutes needed by the computer to reformat the acquired data, so that a total of 15 to 21 minutes elapsed between data acquisition and obtaining a volume measurement.

The true cast volumes and those measured by three dimensional echocardiography are listed in the table. The casts had a median

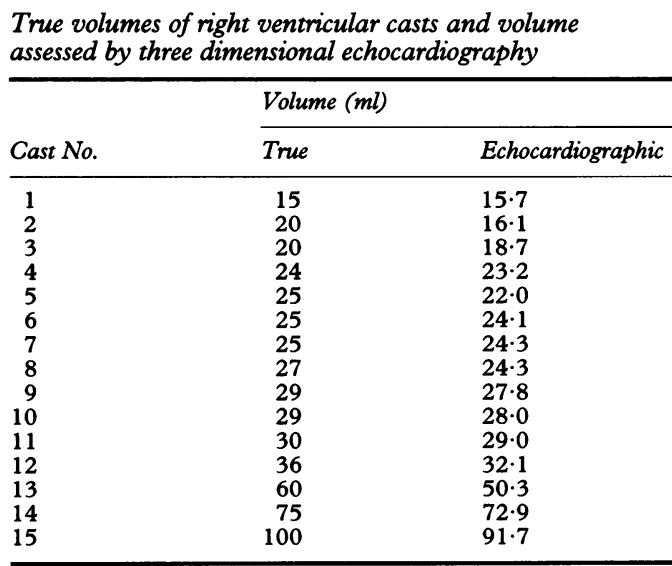


Figure 2 Correlation curve between true volumes of right ventricular casts and volumes measured by three dimensional echocardiography

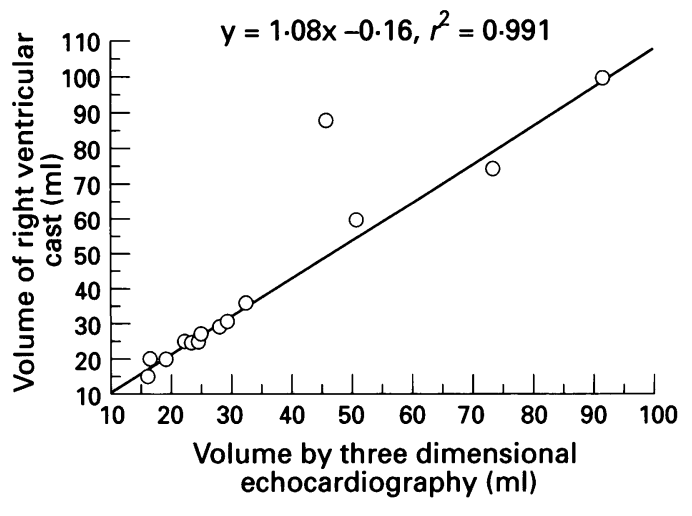

volume of $31 \cdot 1(23) \mathrm{ml}$ (range 15-100) $\mathrm{mls}$ whereas three dimensional echocardiography gave measurements of $29.0(21.7)(15.7-$ $91.7) \mathrm{ml}$. Correlation between true cast volumes and volumes measured by three dimensional echocardiography was 0.99 ( $\mathrm{y}=$ $1.08 \times-0.16$ ) with a SEE of $2.7 \mathrm{ml}$. Figure 2 shows the correlation curve. The limits for agreement between both methods were $-3.1 \mathrm{ml}$ and $8.3 \mathrm{ml}$. In 14 of the 15 measurements the volume by three dimensional echocardiography was smaller than the true volume, with the mean difference being $7 \cdot 4 \%(2 \cdot 8 \%-19 \cdot 5 \%)$. Interobserver variability was $4.5 \%(0.4 \%-13.6 \%)$ and intraobserver variability $4.3 \%(0 \cdot 2 \%-9 \cdot 3 \%)$. The right ventricular volume of the normal heart was $3.2 \mathrm{ml}$; the volume measured by three dimensional echocardiography was $3.5 \mathrm{ml}$.

\section{Discussion}

Our data show that right ventricular volume can be accurately and reproducibly assessed in vitro using a commercially available three dimensional echocardiographic technique. This method of assessing right ventricular volumes would be time consuming, however, if right ventricular function were evaluated by calculating end systolic and diastolic volumes (it would take about 40 minutes). Previous studies have shown that determining right ventricular volume by three dimensional echocardiography with a different means of data acquisition is accurate. ${ }^{812}$ The important difference in our study is that we used casts of human ventricles with an irregular interior surface rather than balloons inflated inside the right ventricle. Although we measured only one normal heart, we could detect no difference in accuracy of three dimensional echocardiographic volume measurements with an interface of fluid and human tissue rather than an interface of fluid and silicone. Although the speed of sound in silicone is different from that in mammalian tissues, which may affect image resolution, this effect on resolution is mainly a problem in solid structures. ${ }^{13} \mathrm{We}$, however, used hollow casts filled with water, which should not have substantially affected the volumes measured. Our data show that volumes of complex geometrical shapes can be assessed in vitro by three dimensional echocardiography. Obviously, assessing the three dimensional volume of a complex geometric structure such as a right ventricle is difficult when using simplified formulas and areas.

Cross sectional angiographic studies have produced only limited correlations with other imaging modes or true right ventricular volumes. ${ }^{14}$ Furthermore, these geometric assumptions may be invalid in right ventricles, whose shape is further distorted by abnormal volume or pressure loading. Echocardiography allows repeated measurements to be made non-invasively. The technique of three dimensional reconstruction by echocardiography should also be able to take account of variations in right ventricular anatomy - for example, in patients with atrial septal defect, anomalous pulmonary venous drainage, or pulmonary incompetence after repair of tetralogy of Fallot, after transposition of the great arteries in arterial redirection (Mustard or Senning) procedures, or in congenitally corrected transposition. The last three groups of patients may develop important right ventricular dysfunction, and paediatric cardiologists need to have a reliable, preferably non-invasive, tool to assess right ventricular function serially.

Nuclear magnetic resonance imaging is also non-invasive and provides accurate assessments of right ventricular volume. ${ }^{15}$ It measures volume in a similar way to three dimensional echocardiographic tomography. Magnetic resonance imaging has previously been compared to three dimensional echocardiography in the assessment of left ventricular volumes and was considered to be the reference standard. ${ }^{16}$ However, it is not as widely available as echocardiography and in some countries is more expensive. The system of obtaining three dimensional echocardiographic data that we used in this study is commercially available and easy to use in a clinical setting, albeit very time consuming. ${ }^{67}$ Previous experience has shown that data acquisition of multiple sequential cross sections of the heart using the Tomtec system takes two to five minutes and can be achieved in most patients in the paediatric age group through a transthoracic or subcostal window, especially when rotational or fan-like approaches of acquiring data suitable for three dimensional reconstruction are used. ${ }^{617}$ Of course, to measure volume accurately the whole (right) ventricle must be imaged without dropout at the endocardium and blood interphase. Previous studies assessing right ventricular volumes by three dimensional echocardiography found comparable accuracy to our method but the measurement required a complex system of data acquisition using a spatial locator. ${ }^{12}$ One study using the spatial locator measured the volumes of a balloon in the right ventricle, ${ }^{8}$ which smoothed out the right ventricular trabeculations, whereas the casts of human ventricles used in our study had irregular interior surfaces.

An important finding in our in vitro study was that three dimensional echocardiography tended to slightly underestimate right ventricular volumes. This may be because of limita- 
tions in lateral resolution of the mechanical ultrasound transducer or because the algorithms we used to format the parallel cross sections into a three dimensional data cube result in thickening of structures. Although the individual slices of the volume data, whose area was manually outlined were displayed like a cross sectional image of the cast, the free walls of the casts in these displays were thickened and may have influenced the volume calculations. As all structures including the endocardium generally appear thickened, we rarely encountered artifactual dropouts, and poor visualisation of the endocardium and blood interface is rarely a problem in clinical studies. Despite the drawback that the interior surface of the casts was thickened, we could still obtain reliable and reproducible measurements of right ventricular volume. Future work entails applying this technique in patients with normal right ventricles and those with either volume or pressure overload or a combination of both. With transthoracic or subcostal data acquisition one potential problem is that it may be difficult to include the whole of the right ventricle in the dataset. Recently the use of rotational ${ }^{17}$ rather than parallel scanning, as previously described by us in a clinical setting, ${ }^{7}$ has improved data acquisition, especially through a subcostal or apical window. Furthermore, we, like others, have begun to acquire multiple sequential cross sections of the heart suitable for three dimensional reconstruction with a multiplane transoesophageal probe. ${ }^{18}$ This approach is, however, limited to patients weighing more than $20 \mathrm{~kg}$. The probe we used in our clinical studies has a maximal diameter of $15.7 \mathrm{~mm}$ and thus is not suitable for use in infants and smaller children. In addition, so far we have acquired data for three dimensional reconstruction with a multiplane transoesophageal probe exclusively in patients under general anaesthesia, so that the clinical use of this method is limited.

We conclude that the system of three dimensional echocardiography described in this study can reliably and reproducibly assess in vitro volumes of right ventricles of different shapes. Future clinical studies need to be performed to examine the clinical usefulness of three dimensional echocardiography in assessing right ventricular volumes in vivo.
MV was supported by a grant from the European Society of Cardiology; PAW was supported in part by the Scott Rhodes Research Fund.

1 Graham TP, Jarkamani JM, Atwood GF, Cannent RV Right ventricular volume determination in children normal values and observations with volume or pressure overload. Circulation 1973;47:144-53.

2 Silverman NH, Hudson S. Evaluation of right ventricular volume and ejection fraction in children by twodimensional echocardiography. Pediatr Cardiol 1983;4: 197-203.

3 Fisher EA, Dubrow JW, Hastreiter AR. Right ventricular volume in congenital heart disease. Am $\mathcal{F}$ Cardiol 1975; 36:67-75.

4 Levine RA, Gibson TC, Arez T, Gillam LD, Guyer DE, King ME, et al. Echocardiographic measurement of ring ME, et al. Echocardiographic measurement of

5 Wollschläger H, Zeiher AM, Klein HP, Kasper W, Geibel A, Wollschläger S. Transesophageal echo computer A, Wollschläger S. Transesophageal echo computer tomography: a new method for dynamic 3-D im

6 Vogel M, Lösch S. Dynamic three-dimensional echocardiography with a computed imaging probe: initial clinical experience with transthoracic application in infants and children with congenital heart defects. Br Heart f 1994;71:462-7.

7 Pandian NG, Roelandt J, Nanda NC, Sugeng L, Cao Q-L, Azevedo J, et al. Dynamic three-dimensional echocardiography: methods and clinical potential. Echocardiography 1994;11:237-59.

8 Jiang L, Siu SC, Handschuhmacher MD, Guererro JL, Vasquez de Prada JA, King ME, et al. Threedimensional echocardiography. In vivo validation for right ventricular volume and function. Circulation 1994; 89:2342-50.

9 Vogel M, Ho SY, Anderson RH. Comparison of threedimensional echocardiographic findings with anatomic specimens of various congenitally malformed hearts. $B r$ Heart $\mathcal{f}$ (in press)

10 Marx GR, Fulton DR, Pandian NG, Vogel M, Cao Q-L, Ludomirsky A, et al. Delineation of site, relative size and dynamic geometry of atrial septal defects by real-time three-dimensional echocardiography. $\Im \mathrm{Am}$ Coll Cardiol 1995;25:482-91.

11 Bland M, Altman DG. Statistical methods for assessing agreement between two methods of clinical measurement. Lancet 1986;i:307-10.

12 Linker DT, Moritz WE, Pearlman AS. A new threedimensional echocardiographic method of right ventricular volume measurement: in vitro validation. $\mathcal{f} \mathrm{Am}$ Coll Cardiol 1986;8:101-6.

13 Madson EL, Zagzegbski JA, Banjavie RA, Jutila RE. Tissue mimicking materials for ultrasound phantoms. Tissue mimicking mate

14 Arcilla RA, Tsai P, Thilenius $O$, Ranniger $K$. Angiographic method for volume estimation of right and left ventricles. Chest 1971;60:446-54

15 Helbing WA, Rebergen SA, Malieppaard C, Ottenkamp J, DeRoos A. Assessment of right ventricular volumes in children by multisection gradient echo magnetic resonance imaging [abstract]. Eur Heart $\mathcal{f}$ 1994;15 (suppl): 460 .

16 Gopal A, King DL, Katz J, Boxt LM, King DL Jr, Shao MYC. Three-dimensional echocardiographic volume computation by polyhedral surface reconstruction: in vitro validation and comparison to magnetic resonance imaging. $¥ \mathrm{Am}$ Soc Echocardiogr 1992;5:115-24.

17 Ludomirsky A, Vermillion R, Nesser J, Marx G, Vogel M, Derman R, et al. Transthoracic real time 3-dimensional echocardiography using rotational scanning approach for data acquisition. Echocardiography 1994;11:599-606.

8 Martin RM, Graham MM, Kao R, Bashein G. Measurement of left ventricular ejection fraction and
volumes with three-dimensional reconstructed transvolumes with three-dimensional reconstructed trans-
esophageal ultrasound scans: comparison to radionuclide and thermal dilution measurements. fournal of Cardiothoracic Anesthesia 1989;3:260-8. 